\title{
INTERSPERSIONS AND DISPERSIONS
}

\author{
CLARK KIMBERLING
}

(Communicated by William W. Adams)

\begin{abstract}
An array $A=\left(a_{i j}\right)$ of all the positive integers is an interspersion if the terms of any two rows, from some point on, alternate in size, and a dispersion if, for a suitable sequence $\left(s_{n}\right)$, the recurrence $a_{j}=s_{a_{j-1}}$ holds for each entry $a_{j}$ of each row of $A$, for $j \geq 2$. An array is proved here to be an interspersion if and only if it is a dispersion. Such arrays whose rows satisfy certain recurrences are considered.
\end{abstract}

\section{Definitions}

An array $A=\left(a_{i j}\right), i \geq 1, j \geq 1$, of positive integers is anterspersion if

(I1) the rows of $A$ comprise a partition of the positive integers;

(I2) every row of $A$ is an increasing sequence;

(I3) every column of $A$ is an increasing (possibly finite) sequence;

(I4) if $\left(u_{j}\right)$ and $\left(v_{j}\right)$ are distinct rows of $A$, and if $p$ and $q$ are any indices for which $u_{p}<v_{q}<u_{p+1}$, then $u_{p+1}<v_{q+1}<u_{p+2}$.

Property (I4) motivates the name interspersion as follows. Suppose $\left(u_{j}\right)$ and $\left(v_{j}\right)$ are distinct rows of $A$. Assume without loss that $u_{1}<v_{1}$, and let $h$ be the index satisfying $u_{h}<v_{1}<u_{h+1}$. Then (I4) inductively implies that

$$
u_{h}<v_{1}<u_{h+1}<v_{2}<u_{h+2}<v_{3}<u_{h+3}<v_{4}<u_{h+4}<\cdots \text {. }
$$

That is, given any two rows of $A$, beginning at the first term of the row having the greater initial term, all the following terms are individually separated by individual terms of the other row. See Tables 1 and 2 in $\S 3$ for numerical examples of interspersions.

An array $B=\left(b_{i j}\right)$ is a dispersion [specifically the dispersion of the sequence $\left.\left(s_{k}\right)\right]$ if

(D1) the first column of $B$ is a strictly increasing sequence, and there exists a strictly increasing sequence $\left(s_{k}\right)$ such that

(D2) $b_{12}=s_{1} \geq 2$,

(D3) the complement of the set $\left\{b_{i 1}: i \geq 1\right\}$ is the set $\left\{s_{k}\right\}$,

(D4) $b_{i j}=s_{b_{i, j-1}}$ for all $j \geq 3$ for $i=1$ and for all $j \geq 2$ for all $i \geq 2$.

Received by the editors February 6, 1990 and, in revised form, October 1, 1990 and June 17, 1991.

1991 Mathematics Subject Classification. Primary 11B75; Secondary 11 B37.

Key words and phrases. Interspersion, dispersion, Stolarsky array, recurrence. 
Such an arrary $B$ disperses the sequence $\left(s_{k}\right)$ in the sense that the rows of the array remaining after deleting from $B$ its first column comprise a partition of the set $\left\{s_{k}\right\}$. We shall prove that every interspersion is a dispersion (Theorem 1) and that every dispersion is an interspersion (Theorem 2). Then we shall examine several examples of such arrays, with a particular interest in those whose rows are linear recurrence sequences. Finally, we shall state an open question concerning sequences that are capable of being the first row of an interspersion, all of whose row sequences satisfy a simple recurrence relation.

\section{EQUIVALENCE OF INTERSPERSION AND DISPERSION}

Lemma 1. Suppose $A=\left(a_{i j}\right)$ is an interspersion. For $i \geq 1$ and $j \geq 1$, let $r=a_{i, j+1}-a_{i j}$. Then each of the $r$ numbers $a_{i j}+1, a_{i j}+2, \ldots, a_{i, j+1}$ lies in one and only one row of $A$ numbered from 1 up to $r$.

Proof. First, if $r=1$ then $i=1$, for if not, then $a_{i, j+1}-a_{i j}=1$ for some $i \geq 2$ and $j \geq 1$, but then by Property (I4) some number $x$ in row 1 satisfies $a_{i j}<x<a_{i, j+1}$, contrary to $r=1$.

Now $a_{i, j+1}$ lies in row $i$. If $r=1$ then $i=1$, and the proof is finished.

So suppose $r \geq 2$, and let $m$ be any positive integer satisfying $1 \leq m \leq r$ and $m \neq i$, and assume that $a_{m 1}<a_{i, j+1}$. By Property (I4), there is an index $p$ satisfying $a_{i j}<a_{m p}<a_{i, j+1}$, so that $a_{m p}=a_{i j}+h$ for some $h$ satisfying $1 \leq h \leq r-1$. That is, if the first term of row $m$ is less than $a_{i, j+1}$ then row $m$ contains a number $a_{i j}+h$, where $1 \leq h \leq r-1$. We shall show next that such a row contains only one such number.

Suppose

$$
a_{m p}=a_{i j}+h \quad \text { and } a_{m q}=a_{i j}+k,
$$

with $h<k$, are two of the numbers in question, both lying in row $m$. We have $a_{m p}<a_{i, j+1}<a_{m, p+1}$ by (I4) so that $a_{m p}<a_{m q}<a_{m, p+1}$. However, it is impossible for $a_{m q}$ to lie between two consecutive terms of row $m$. This contradiction shows that the $r-1$ numbers lie, one per row, in the first $r$ rows of $A$. Note that this means that our assumptions $1 \leq m \leq r, m \neq i$, and $a_{m 1}<a_{i, j+1}$ were sufficient to account for all of the $r-1$ numbers.

We have to show also that $i \leq r$; i.e., that $a_{i, j+1}$ lies in one of the first $r$ rows of $A$. By (I4), each of the rows numbered from 1 to $i-1$ contains a number $x$ satisfying $a_{i j}<x<a_{i, j+1}$. By (I1) these $x$ are distinct, so there are $i-1$ of them. As there are only $r-1$ distinct positive integers between $a_{i j}$ and $a_{i, j+1}$, we conclude that $i \leq r$.

Finally, if $a_{m 1}>a_{i, j+1}$ for some $m$ satisfying $1 \leq m \leq r$, then by (I3), $a_{m+n, 1}>a_{i, j+1}$ for all $n \geq 0$, so by (I2), no element of any of the rows numbered $m+n$, for $n \geq 0$, is $<a_{i, j+1}$. Then the array $A$ does not contain all the integers $a_{i j}+h$ for $1 \leq h \leq r$, contrary to (I1).

Lemma 2. If $A=\left(a_{i j}\right)$ is an interspersion, then

$$
a_{i, j+1}=a_{i j}+C_{1}^{*}\left(a_{i, j+1}\right),
$$

where $C_{1}^{*}(m)$ denotes, for $m \geq 1$, the number of terms in the first column of $A$ that are $\leq m$. 
Proof. Let $i$ and $j$ be arbitrary positive integers, and write $r=a_{i, j+1}-a_{i j}$.

Case 1. Suppose $r=1$. Then $i=1$, as in the proof of Lemma 1 . Now if $a_{1, j+1}-a_{1 j}=1$ for all $j$, then row 1 is simply the sequence $(j)$ of positive integers, and $A=(j)$, so that $C_{1}^{*}\left(a_{1, j+1}\right)=1$ as required.

Otherwise, let $K$ be the least $j$ for which $a_{1, j+1}-a_{1 j} \geq 2$. Then $C_{1}^{*}\left(a_{1 j}\right)=1$ for all $j \leq K$, and we need only show that there are no other pairs $(i, j)$ for which $r=1$. The number $a_{1 K}+1$ lies in row $p$ for some $p \geq 2$. Write $a_{1 K}+1=a_{p q}$, so that $a_{1 K}<a_{p q}<a_{1, K+1}$, and by (I4),

$$
a_{p q}<a_{1, K+1}<a_{p, q+1}<a_{1, K+2}<\cdots \text {. }
$$

That is, $a_{1, j+1}-a_{1 j} \geq 2$ for all $j \geq K$. Clearly, (I2) and (I3) force $(p, q)=$ $(2,1)$, and it follows that $a_{i, j+1}-a_{i j} \geq 2$ for all $j \geq 1$ for all $i \geq 2$. Thus, all pairs $(i, j)$ for which $r=1$ have been considered.

Case 2. Suppose $r \geq 2$. By Lemma 1, row $r$ contains a number $x$ satisfying $x \leq a_{i, j+1}$ and no higher numbered row contains such a number. Since, by (I2), $a_{r 1} \leq x \leq a_{i, j+1}$, we conclude by (I4) that $C_{1}^{*}\left(a_{i, j+1}\right)=a_{i, j+1}-a_{i, j}$.

Lemma 3. Suppose $A=\left(a_{i j}\right)$ is an interspersion. Let $\left(s_{k}\right)$ be the sequence obtained by arranging in increasing order the numbers in the complement of the set $\left\{a_{i 1}: i \geq 1\right\}$. Suppose $i \geq 1$ and $j \geq 1$. Then $a_{i, j+1}=s_{a_{i, j}}$.

Proof. First, we consider the first two terms in any row: suppose $i$ is arbitrary and $j=1$. By Lemma 2, the number of elements of column 1 that are $\leq a_{i 2}$ is $a_{i 2}-a_{i 1}$. Thus, the number of numbers of the sequence $\left(s_{k}\right)$ that are $\leq a_{i 2}$ is $a_{i 1}$, which is to say that $a_{i 2}=s_{a_{i, 1}}$.

Continuing with arbitrary $i$, let $j \geq 2$, and let $S$ be the set of numbers in $\left(s_{k}\right)$ that are $\leq a_{i, j+1}$. As an induction hypothesis, suppose that $a_{i, j-1}$ of these numbers are $\leq a_{i j}$. Then to prove $a_{i, j+1}=s_{a_{i, j}}$, we must prove that the number of numbers in the set

$$
T=\left\{a_{i j}+1, a_{i j}+2, \ldots, a_{i, j+1}\right\}
$$

that lie in $S$ is $a_{i j}-a_{i, j-1}$.

To that end, note that by Lemma 1 , the $a_{i, j+1}-a_{i j}$ numbers in $T$ lie, one per row, in rows numbered from 1 to $a_{i, j+1}-a_{i j}$. The induction hypothesis implies that the rows numbered from 1 to $a_{i j}-a_{i, j-1}$ all have column 1 entries that are $\leq a_{i j}$, so that $a_{i j}-a_{i, j-1}$ of the numbers in $T$, by Property (I2), do not lie in column 1. All the other numbers in $T$ do lie in column 1 , because of (I2). Therefore $a_{i j}-a_{i, j-1}$ is the number of numbers in $T$, as was to be shown.

Theorem 1. If an array $A=\left(a_{i j}\right)$ satisfies Properties (I1)-(I4) then it satisfies Properties (D1)-(D4).

Proof. Suppose $A$ satisfies (I1)-(I4). Let $\left(s_{k}\right)$ be the sequence obtained by arranging in increasing order the numbers in the complement of the set $\left\{a_{i 1}: i \geq\right.$ $1\}$. We shall construct the dispersion $B=\left(b_{i j}\right)$ of the sequence $\left(s_{k}\right)$ and then prove that $B=A$.

Let $b_{11}=1$ and $b_{12}=s_{1}$. Define $b_{1 j}$ recursively as $s_{b_{1, j-1}}$ for all $j \geq 3$. Let $b_{21}$ be the least positive integer not in $\left\{b_{1 j}\right\}$, and define $b_{2 j}=s_{b_{2, j-1}}$ for all $j \geq 2$. Once the $(i-1)$ st row is constructed for arbitrary $i \geq 3$, let $b_{i 1}$ be the least positive integer not in $\left\{b_{h j}: h \leq i-1\right\}$ (unless there are no such integers, in which case clearly $A$ and now $B$ consist of only finitely many rows), and 
define $b_{i j}=s_{b_{i, j-1}}$ for all $j \geq 2$. It is clear that the array $B$ so defined satisfies (D1)-(D4) and is the only such array for the particular sequence $\left(s_{k}\right)$.

Suppose for arbitrary $i$ that $b_{i 1}=a_{i 1}$, and as an induction hypothesis, assume that $b_{i j}=a_{i j}$. Then by Lemma 3 ,

$$
b_{i, j+1}=s_{b_{i, j}}=s_{a_{i, j}}=a_{i, j+1} \text {. }
$$

Thus, if the initial terms $b_{i 1}$ and $a_{i 1}$ are equal, then the entire rows $\left(b_{i j}\right)$ and $\left(a_{i j}\right)$ are equal. It remains to be seen that $b_{i 1}=a_{i 1}$ for all $i \geq 1$.

First, $b_{11}=a_{11}$, by definition of $b_{11}$. Assume that $b_{i 1}=a_{i 1}$ for all $i \leq k$, where $k$ is an arbitrary positive integer. As already shown, the sets $B^{\prime}=$ $\left\{b_{h j}: h \leq k, j \geq 1\right\}$ and $A^{\prime}=\left\{a_{h j}: h \leq k, j \geq 1\right\}$ are equal. The number $b_{k+1,1}$ is the least positive integer not in $B^{\prime}$, hence the least not in $A^{\prime}$. By (I2), $b_{k+1,1}$ must be the first number in its row in the truncated array $A-A^{\prime}$, and by (I3), this number must therefore occupy the position $(k+1,1)$ in $A$. We conclude that $b_{k+1,1}=a_{k+1,1}$ and by induction that $b_{i 1}=a_{i 1}$ for all $i \geq 1$, as was required to finish the proof.

Theorem 2. If an array $A=\left(a_{i j}\right)$ satisfies Properties (D1)-(D4), then it satisfies Properties (I1)-(I4).

Proof. First, suppose $A$ satisfies (D1)-(D4), and let $\left\{s_{k}\right\}$ be as in (D2)-(D4). Property (D4) shows that row $i$ is a strictly increasing sequence for all $i \geq 1$, so that (I2) holds.

Next, we shall see that every positive integer $m$ is a term of $B$. If not, let $n$ be the least not in $B$. Then $n$ cannot lie in column 1 , so by (D3), $n=s_{m}$ for some $m$, and $m<n$ since $\left(s_{k}\right)$ is strictly increasing. But then $m=b_{i j}$ for some $i$ and $j$. By (D4), $n=b_{i, j+1}$, a contradiction.

To see that each $m$ occurs in $B$ only once, we recognize three cases: (1) if $b_{h 1}=b_{i 1}$ then $h=i$, by (D1); (2) if $b_{h 1}=b_{i j}$, where $j \geq 2$, then $b_{h 1}=s_{b_{1, j-1}}$, contrary to (D3); (3) suppose $b_{h k}=b_{i j}$, where $j \geq 2, k \geq 2$, and $(i, j) \neq(h, k)$. Assume $k$ is the least positive integer for which $b_{h k}=b_{i j}$ for some choice of $h, i$, and $j$. Then since $b_{h k}=s_{b_{h, k-1}}$ and $b_{i j}=s_{b_{i, j-1}}$ and $\left(s_{k}\right)$ is strictly increasing, we have $b_{h, k-1}=b_{i, j-1}$, a contradiction. We conclude that every positive integer occurs once and only once in $B$, so that (I1) holds.

Now (D1) implies $a_{i+1,1}>a_{i, 1}$ for all $i \geq 1$. For any fixed $i \geq 1$, assume for arbitrary $j \geq 1$ that $a_{i+1, j}>a_{i, j}$. Then

$$
a_{i+1, j+1}=s_{a_{i+1, j}}>s_{a_{i, j}}=a_{i, j+1},
$$

since $\left(s_{k}\right)$ is a strictly increasing sequence. Thus, by induction on $j, a_{i+1, j+1}>$ $a_{i, j+1}$ for all $j \geq 1$, and since this holds for all $i$, we conclude that column $j$ is strictly increasing. Thus, (I3) holds.

Finally, suppose $\left(u_{j}\right)$ and $\left(v_{j}\right)$ are distinct rows of $A$ and that $p$ and $q$ are indices for which $u_{p}<v_{q}<u_{p+1}$. Then $s_{u_{p}}<s_{v_{q}}<s_{u_{p+1}}$, since $\left(s_{k}\right)$ is strictly increasing. By (D4) $u_{p+1}<v_{q+1}<u_{p+2}$, so that (I4) holds, and a proof of Theorem 2 is completed.

The concepts of interspersion (of the rows) and dispersion (of a sequence) as defined at the beginning of this paper have now been proved equivalent. In the sequel, any array that is an interspersion will be called an ID array. 
Very simple ID arrays are given by $a_{i j}=i+(j-1) m$ for $i=1,2, \ldots, m$ and all $j \geq 1$, where $m$ is a fixed positive integer. Here $a_{i, j+1}-a_{i j}=m$ for all $i$ and $j$, and the array consists of only $m$ rows. More generally, as can easily be verified, an ID array has infinitely many rows if and only if the differences $a_{1, j+1}-a_{1 j}$ in row 1 are unbounded. When this is the case, we note that the ID array has a natural sort of inverse, defined as follows.

\section{INVERSE ID ARRAYS}

Suppose $A=\left(a_{i j}\right)$ is an ID array containing infinitely many rows. The inverse, $A^{-1}$, of $A$, is the dispersion of the sequence $\left(a_{i 1}\right), i \geq 2$ (i.e., the first column of $A$, minus its first term, 1). With $\left(s_{k}\right)$ as in (D1)-(D4), we write $s^{j}(x)$ for $s\left(s^{j-1}(x)\right)$, where $s^{0}(x) \equiv x$ and $s^{1}(x)=s_{x}$. Let $\left(t_{k}\right)$ be the ordered complement of the set $\left\{s_{k}\right\}$. Then for $A=\left(a_{i j}\right)$ and $A^{-1}=\left(b_{i j}\right)$ we can write $a_{i j}=s^{j-1}\left(t_{i}\right)$ for all $i \geq 1$ and $j \geq 1$, and $b_{i j}=t^{j-1}\left(s_{i-1}\right)$ for $j \geq 1$ for $i \geq 2$; the first row of $B$ is given by $b_{11}=1$ and $b_{1 j}=t^{j-2}\left(t_{2}\right)$ for $j \geq 2$.

Among ID arrays are those that disperse a strictly increasing sequence $\left(s_{k}\right)$ of the form $s_{k}=[\alpha k+\gamma]$, where $\alpha$ is irrational, $\gamma$ real and $<1$, and $s_{1} \geq 2$. Here, $[x]$ denotes, as usual, the greatest integer $\leq x$. Let $\beta=\alpha /(\alpha-1)$ and $\delta=\gamma /(1-\alpha)$, and assume that $\gamma$ is chosen so that $n \beta+\delta$ is not an integer for any integer $n$. It follows immediately from Fraenkel [1, Theorem XI] that the ordered complement of $\left(s_{k}\right)$ is conveniently given by $t_{k}=[\beta k+\delta]$. See also Graham [2].

As a specific example of such an ID array and its inverse, let $\alpha=(1+\sqrt{5}) / 2$ and $\gamma=\frac{1}{2}$, so that $s_{k}$ represents the integer nearest $\alpha k$ (Tables 1 and 2). The array $A$ is then the Stolarsky array, discussed by Morrison [4], who cites several other references to the Stolarsky array.

The first of these numerical examples also shows that a certain sequence, in this case the Fibonacci numbers, can serve as the first row of an ID array in which all the subsequent rows satisfy a common recurrence relation, in this case, $a_{i j}=a_{i, j-1}+a_{i, j-2}$.

TABLE 1. The Stolarsky array

\begin{tabular}{|c|c|c|c|c|c|c|c|c|}
\hline 12 & 3 & 5 & 8 & 13 & 21 & 34 & 55 & 89 \\
\hline 46 & 10 & 16 & 26 & 42 & 68 & 110 & 178 & 288 \\
\hline 711 & 18 & 29 & 47 & 76 & 123 & 199 & 322 & 521 \\
\hline 915 & 24 & 39 & 63 & 102 & 165 & 267 & 432 & 699 \\
\hline 1219 & 31 & 50 & 81 & 131 & 212 & 343 & 555 & 898 \\
\hline 1423 & 37 & 60 & 97 & 157 & 254 & 411 & 665 & 1076 \\
\hline 1728 & 45 & 73 & 118 & 191 & 309 & 500 & 809 & 1309 \\
\hline 2032 & 52 & 84 & 136 & 220 & 356 & 576 & 932 & 1508 \\
\hline 2236 & 58 & 94 & 152 & 246 & 398 & 644 & 1042 & 1686 \\
\hline 2540 & 65 & 105 & 70 & 275 & 445 & 72 & 165 & 18 \\
\hline
\end{tabular}


TABLE 2. Inverse of the Stolarsky array

$\left.\begin{array}{rrrrrrrrrr}1 & 4 & 12 & 33 & 88 & 232 & 609 & 1596 & 4180 & 10945\end{array}\right]$

\section{First Row AND ITERATION SEQUENCES}

The remainder of this paper addresses the following questions. What sequences $\left(a_{j}\right)$ can be the first row of an ID array $A$ ? And, in the case that such a sequence $\left(a_{j}\right)$ satisfies a recurrence relation $\mathscr{R}$, when is it possible for all the rows of such an ID array $A$ to satisfy $\mathscr{R}$ ?

Theorem 3. A sequence $\left(a_{j}\right)$ of positive integers is the first row of an ID array $A=\left(a_{i j}\right)$ if and only if $a_{1}=1, a_{2} \geq 2$, and $a_{j} \geq 2 a_{j-1}-a_{j-2}$ for all $j \geq 3$.

Proof. The inequality $a_{j} \geq 2 a_{j-1}-a_{j-2}$ can be written as

$$
a_{j}-a_{j-1} \geq a_{j-1}-a_{j-2} \text {. }
$$

If $A$ is an ID array, then $a_{1}=1, a_{2} \geq 2$, and (1) follows immediately from Lemma 2 since the first column of $A$ is strictly increasing.

Now assume $a_{1}=1, a_{2} \geq 2$, and (1) for all $j \geq 3$, and construct an array $\left(a_{i j}\right)$ as follows. Let $a_{1 j}=a_{j}$ for all $j \geq 1$. If $a_{j+1}-a_{j}=1$ for all $j$, then the construction is finished, and $A$ is simply the sequence of positive integers.

Step 1. Otherwise, let $k$ be the least $j$ for which $a_{j+1}-a_{j} \geq 2$. Define $a_{21}=a_{k}+1, a_{31}=a_{k}+2, \ldots$, and $a_{m 1}=a_{k+1}-1$, where $m=a_{k+1}-a_{k}-1$. Now every integer from 1 to $a_{k+1}$ is positioned in the array being constructed, and clearly, for all of row 1 and for $\left(a_{i 1}\right), 1 \leq i \leq m$, Properties (I2)-(I4), modified in the obvious way to apply to the partial array, hold.

Step 2. For $j=k+1$, we have by (1) at least as many integers $a_{j}+1, a_{j}+$ $2, \ldots, a_{j+1}-1$ to position as in Step 1. Define $a_{22}=a_{j}+1, a_{32}=a_{j}+2, \ldots$, and $a_{m 2}=a_{j+1}-1$, where $m=a_{j+1}-a_{j}-1$. If $a_{j+1}-a_{j}=a_{k+1}-a_{k}$, then every integer from 1 to $a_{j+1}$ is positioned in $A$, and Step 2 is finished. Otherwise, assign the remaining $a_{j+1}-a_{j}-\left(a_{k+1}-a_{k}\right)$ integers in increasing order to consecutive positions in column 1 beginning at position $(m+1,1)$. This finishes Step 2. Clearly, Properties (I1)-(I4) suitably restricted hold. We continue inductively:

Step $j$. For arbitrary $j \geq k+1$, position the integers $a_{j}+h$ for $h=$ $1,2, \ldots, a_{j+1}-a_{j}-1$ by placing $a_{j}+h$ at the end of row $h+1$. By this, we 
mean appending $a_{j}+h$ in the next position to the right of the number assigned to row $h+1$ in Step $j-1$, or, if $h$ is large enough that no number was assigned to row $h+1$ in Step $j-1$, then we begin row $h+1$ with $a_{h+1,1}=a_{j}+h$.

It is clear that every positive integer is eventually assigned a position in the array, so that (I1) holds. Moreover, since (I2), (I3), and (I4), suitably restricted, hold after each step of the construction, they hold for the completed construction.

Corollary. Call a sequence $\left(a_{j}\right)$ of positive integers an iteration sequence if there exists a strictly increasing sequence $\left(s_{k}\right)$ of positive integers such that

(i) $a_{1}=s_{1} \geq 2$, and

(ii) $a_{j}=s_{a_{j-1}}$ for $j=2,3, \ldots$.

Then a necessary and sufficient condition that $\left(a_{j}\right)$ be an iteration sequence is that

$$
a_{j} \geq 2 a_{j-1}-a_{j-2} \text { for all } j \geq 3 \text {. }
$$

Proof. First suppose $\left(a_{j}\right)$ is an iteration sequence. Let $b_{11}=1$ and $b_{12}=$ $s_{1}=a_{1}$. Let $\left\{b_{i 1}: i \geq 1\right\}$ be the ordered complement of $\left\{s_{k}\right\}$, and define $b_{i j}=s_{b_{i, j-1}}$ for all $j \geq 3$ for $i=1$ and for all $j \geq 2$ for all $i \geq 1$. Then Properties (D1)-(D4) hold, so that the array $B=\left(b_{i j}\right)$ is an ID array. Its first row, by (D4) and (ii), is the sequence $1, a_{1}, a_{2}, a_{3}, \ldots$. By Theorem 3 , statement (2) holds.

For the converse, suppose (2) holds. By Theorem 3, the sequence $1, a_{1}, a_{2}$, $a_{3}, \ldots$ is the first row of an ID array. Let $\left(s_{k}\right)$ be as in Properties (D1)-(D3). Then by (D4), statement (ii) holds, so that $\left(a_{j}\right)$ is an iteration sequence.

(The open question posed in $\S 5$ can be regarded as a question about iteration sequences.)

\section{RECURRENT ROW SEQUENCES}

We return now to the question of ID arrays all of whose rows satisfy a common recurrence relation $\mathscr{R}$. This question appears to be difficult in general. We shall consider only second-order linear recurrences generated by nearly arithmetic sequences of the form $s_{k}=[\alpha k+\gamma]$, where $\alpha>1$ is irrational and $s_{1} \geq$ 2 . To this end, suppose $a$ and $b$ are integers and $x^{2}-a x-b=(x-\alpha)(x-\beta)$, where $\alpha>1$ and $|\beta|<1$. For arbitrary $\gamma$ and positive integer $n$, let

$$
t_{1}=[\alpha n+\gamma], t_{2}=\left[\alpha t_{1}+\gamma\right], \ldots, t_{k}=\left[\alpha t_{k-1}+\gamma\right], \ldots .
$$

We are interested in those $\alpha, \beta$, and $\gamma$ for which, for every $n$, the sequence $\left(t_{k}\right)$ satisfies the recurrence relation

$$
t_{k}=a t_{k-1}+b t_{k-2} \text { for all } k \geq 3,
$$

because in this case the dispersion of $\left(s_{k}\right)$ has all row sequences satisfying (4). In the notation at hand, the dispersion of $\left(s_{k}\right)$, as constructed in the proof of Theorem 1, is as follows:

$$
\begin{aligned}
& b_{11}=1, b_{12}=[\alpha+\gamma], \ldots, b_{1 j}=\left[\alpha b_{i, j-1}+\gamma\right] ; \\
& b_{21}=\text { least positive integer not in }\left\{b_{1 j}\right\}, b_{22}=\left[\alpha b_{21}+\gamma\right], \ldots, \\
& b_{2 j}=\left[\alpha b_{2, j-1}+\gamma\right] ; \\
& b_{31}=\text { least positive integer not in }\left\{b_{1 j}\right\} \cup\left\{b_{2 j}\right\}, \text { and so on. }
\end{aligned}
$$


When all the rows of this dispersion satisfy a recurrence $\mathscr{R}$, we call the dispersion an $\mathscr{R}$ array and say that the sequence $\left(s_{k}\right)$ is recurrence-complete. (More generally, we may speak of the set of $n$ for which the sequence (4) satisfies $\mathscr{R}$ as the recurrence set of $\left(s_{k}\right)$, so that $\left(s_{k}\right)$ is recurrence-complete if its recurrence set is the set of all positive integers.)

Theorem 4. Suppose $\alpha>1$ is irrational and $|\beta|<1$. Suppose $\mathscr{R}$ is the recurrence (4), where $x^{2}-a x-b=(x-\alpha)(x-\beta)$. If $-1<\beta<0$, then the dispersion of the sequence $\left(s_{k}\right)=([\alpha k+\gamma])$ is an $\mathscr{R}$ array if and only if

$$
\frac{\beta}{\beta-1} \leq \gamma \leq \frac{1}{1-\beta} \text {. }
$$

If $0 \leq \beta<1$ then the dispersion of $\left(s_{k}\right)$ is an $\mathscr{R}$ array if and only if $0 \leq \gamma \leq 1$. Proof. For each row of the dispersion of $\left(s_{k}\right)$ there is a positive integer $n$ for which the row begins with $t_{1}=[\alpha n+\gamma]$ and $t_{2}=\left[\alpha t_{1}+\gamma\right]$ and continues inductively with $t_{k}=\left[\alpha t_{k-1}+\gamma\right]$. What we must prove then is that for every $n \geq 1,\left(t_{k}\right)$ satisfies recurrence (4) if and only if the conditions stated above for $\gamma$ hold.

Recall (e.g., Hall [3, p. 22]) that a sequence $\left(t_{k}\right)$ satisfies a recurrence (4) if and only if $t_{k}=c_{1} \alpha^{k}+c_{2} \beta^{k}$ for all $k \geq 1$, where

$$
c_{1}=\frac{t_{2}-\beta t_{1}}{\alpha(\alpha-\beta)} \quad \text { and } \quad c_{2}=\frac{t_{2}-\alpha t_{1}}{\beta(\beta-\alpha)} \text {. }
$$

The following statements, for all $k \geq 2$, hence for all $j \geq 0$, are therefore equivalent:

$$
\begin{aligned}
& t_{k}=\left[\alpha t_{k-1}+\gamma\right]=c_{1} \alpha^{k}+c_{2} \beta^{k}, \\
& {\left[\alpha\left(c_{1} \alpha^{k-1}+c_{2} \beta^{k-1}\right)+\gamma\right] }=c_{1} \alpha^{k}+c_{2} \beta^{k}, \\
& {\left[c_{1} \alpha^{k}+c_{2} \beta^{k}+c_{2} \beta^{k-1}(\alpha-\beta)+\gamma\right] }=c_{1} \alpha^{k}+c_{2} \beta^{k}, \\
& {\left[c_{2} \beta^{k-1}(\alpha-\beta)+\gamma\right] }=0, \\
& 0 \leq\left(\alpha t_{1}-t_{2}\right) \beta^{j}+\gamma<1, \quad 0 \leq \varepsilon_{n} \beta^{j}+\gamma\left(1-\beta^{j}\right)<1,
\end{aligned}
$$

where $\varepsilon_{n}=\alpha[\alpha n+\gamma]+\gamma-[\alpha[\alpha n+\gamma]+\gamma]$ is the fractional part of $\alpha[\alpha n+\gamma]+\gamma$, so that the final equivalent statement can be written as

$$
\frac{\varepsilon_{n} \beta^{j}}{\beta^{j}-1} \leq \gamma \leq \frac{\varepsilon_{n} \beta^{j}-1}{\beta^{j}-1} .
$$

If $-1<\beta<0$, then for all $n \geq 1$ and all $j \geq 1$,

$$
\frac{\varepsilon_{n} \beta^{j}}{\beta^{j}-1} \leq \frac{\varepsilon_{n} \beta}{\beta-1}<\frac{\beta}{\beta-1} \text { and } \frac{\varepsilon_{n} \beta^{j}-1}{\beta^{j}-1} \geq \frac{\varepsilon_{n} \beta-1}{\beta-1}>\frac{1}{1-\beta},
$$

so that (5) implies (6). Moreover, (5) is necessary if (6) is to hold for all pertinent $n$ and $j$; to see this, note that the set of fractional parts $((\alpha n+\gamma))$ is dense in the interval $(0,1)$, so that the numbers $\varepsilon_{n}$ also form a dense set, so that

$$
\sup _{n \geq 1} \frac{\varepsilon_{n} \beta}{\beta-1}=\frac{\beta}{\beta-1} \quad \text { and } \quad \inf _{n \geq 1} \frac{\varepsilon_{n} \beta-1}{\beta-1}=\frac{1}{1-\beta} .
$$


If $0 \leq \beta<1$, then

$$
\frac{\varepsilon_{n} \beta^{j}}{\beta^{j}-1}<0=\sup _{j, n \geq 1} \frac{\varepsilon_{n} \beta^{j}}{\beta^{j}-1} \text { and } \quad \frac{\varepsilon_{n} \beta^{j}-1}{\beta^{j}-1} \geq 1=\inf _{j, n \geq 1} \frac{\varepsilon_{n} \beta^{j}-1}{\beta^{j}-1},
$$

so that for $0 \leq \beta<1$ the inequality $0 \leq \gamma \leq 1$ is necessary and sufficient for (6).

Corollary. Let $\alpha$ and $\mathscr{R}$ be as in Theorem 4. Then the dispersion of the sequence $\left(s_{k}\right)=\left(\left[\alpha k+\frac{1}{2}\right]\right)$ is an $\mathscr{R}$ array.

Open question. Suppose $a$ and $b$ are integers and $x^{2}-a x-b=(x-\alpha)(x-\beta)$, where $\alpha$ is irrational, $\alpha>\frac{3}{2}$, and $|\beta|>1$. Let

$$
a_{1}=\left[\alpha+\frac{1}{2}\right], a_{2}=\left[\alpha a_{1}+\frac{1}{2}\right], \ldots, a_{k}=\left[\alpha a_{k-1}+\frac{1}{2}\right], \ldots
$$

Does there exist an $\mathscr{R}$ array $A$ whose first row is the sequence $1, a_{1}, a_{2}, a_{3}$, $\ldots$, where $\mathscr{R}$ is the recurrence (4)? When such an $A$ exists, to what extent must the ordered complement of the first column of $A$ deviate from the sequence $\left(s_{k}\right)=\left(\left[\alpha k+\frac{1}{2}\right]\right)$ or some other easily formulated sequence?

\section{REFERENCES}

1. A. Fraenkel, The bracket function and complementary sets of integers, Canad. J. Math. 21 (1969), 6-27.

2. R. L. Graham, Covering the positive integers by disjoint sets of the form $\{[n \alpha+\beta]: n=$ $1,2, \ldots\}$, J. Combin. Theory Ser. A 15 (1973), 354-358.

3. M. Hall, Jr., Combinatorial theory, Blaisdell, Waltham, MA, 1967.

4. D. R. Morrison, A Stolarsky array of Wythoff pairs, A Collection of Manuscripts Related to the Fibonacci Sequence, Fibonacci Assoc., Santa Clara, CA, 1980, pp. 134-136.

Department of Mathematics, University of Evansville, Evansville, Indiana 47722 